\title{
Antley-Bixler Syndrome: Surgical Management of Ambiguous Genitalia - A Case Report
}

\author{
E.S. Boia ${ }^{\text {a M.C. Popoiu a }}$ M.Puiu ${ }^{\text {a }}$ C.M. Stanciulescu ${ }^{\text {b V.L. David }}{ }^{\text {a }}$ \\ a Victor Babes University of Medicine and Pharmacy, and b Louis Turcanu Emergency Children's Hospital, \\ Timisoara, Romania
}

\section{Key Words}

Antley-Bixler syndrome $\cdot$ Cytochrome P450 oxidoreductase deficiency $\cdot$ Rare disease $\cdot$ Ambiguous genitalia repair

\begin{abstract}
Objective: To report a case of type 2 Antley-Bixler syndrome (ABS). Subject and Methods: A 3-year-old boy who had been raised male, with facial dimorphism and malformations of both elbows and forearms, was referred to our unit for ambiguous genitalia. Genetic testing confirmed the diagnosis of ABS. A surgical intervention was performed to correct the ambiguous genitalia through a combined perineal and transabdominal approach. Results: The postoperative course was uneventful and the patient was released from the hospital 10 days after the surgery. Conclusion: Repair of the ambiguous genitalia in this patient was possible, but definitive inferences on the benefit of this intervention cannot be made without long-term follow-up. @ $\quad$ @ 2013 S. Karger AG, Basel
\end{abstract}

\section{Introduction}

Antley-Bixler syndrome (ABS) is a rare congenital malformation involving multiple craniofacial, musculoskeletal and urogenital malformations. It was first de- scribed in 1975 by Antley and Bixler [1], and since then fewer than 50 cases have been reported in the literature [2]. ABS is a heterogeneous disorder; two genetically distinctive forms have been observed: type 1 involves mutations in the FGFR2 gene (10q26) without impairment of steroidogenesis, whereas type 2 involves mutations in the gene encoding cytochrome P450 oxidoreductase (POR), an enzyme which plays a direct role in steroidogenesis $[3,4]$. Clinical features include a typical trapezoidal face with craniosynostosis, midface hypoplasia and a flat nose, femoral or humeral bowing, radiohumeral synostosis and multiple joint contractures [5]. Type 2 ABS is an autosomal recessive disorder, and it is associated with abnormal genitalia in both sexes due to impaired steroidogenesis [6]. Occasionally, a diverse range of cardiac, renal, gastrointestinal and vertebral malformations may occur [5]. Recently, we encountered a case of type 2 ABS. This paper reports the clinical particularities of that case and the surgical approach adopted towards management of the ambiguous genitalia.

ABS is a very rare disease and, as far as we know, this is the first report of an ABS patient undergoing surgical treatment for ambiguous genitalia. This case also represents a first instance for Romania; there are no other previous reports of $A B S$ in our country.

\begin{tabular}{ll}
\hline KARGER & $\begin{array}{l}\text { (c) 2013 S. Karger AG, Basel } \\
1011-7571 / 13 / 0234-0384 \$ 38.00 / 0\end{array}$ \\
$\begin{array}{l}\text { E-Mail karger@karger.com } \\
\text { www.karger.com/mpp }\end{array}$ & $\begin{array}{l}\text { This is an Open Access article licensed under the terms of the } \\
\text { Creative Commons Attribution-NonCommercial 3.0 Un- } \\
\text { ported license (CC BY-NC) (www.karger.com/OA-license), } \\
\text { applicable to the online version of the article only. Distribu- } \\
\text { tion permitted for non-commercial purposes only. }\end{array}$
\end{tabular}

Dr. Vlad-Laurentiu David Dr. Iosif Nemoianu No. 2 RO-300011 Timisoara (Romania) E-Mail david.vlad@yahoo.com 


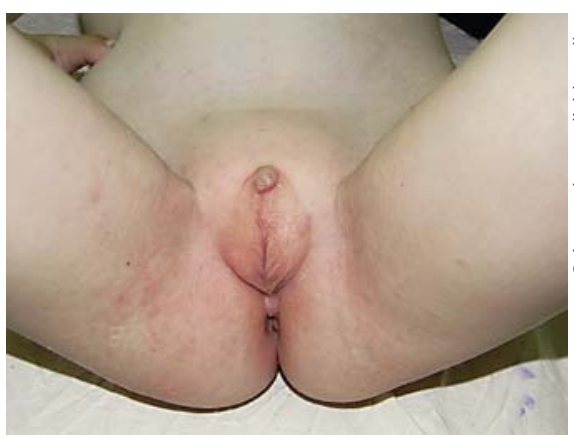

Fig. 1. Hypoplastic penis-like structure.
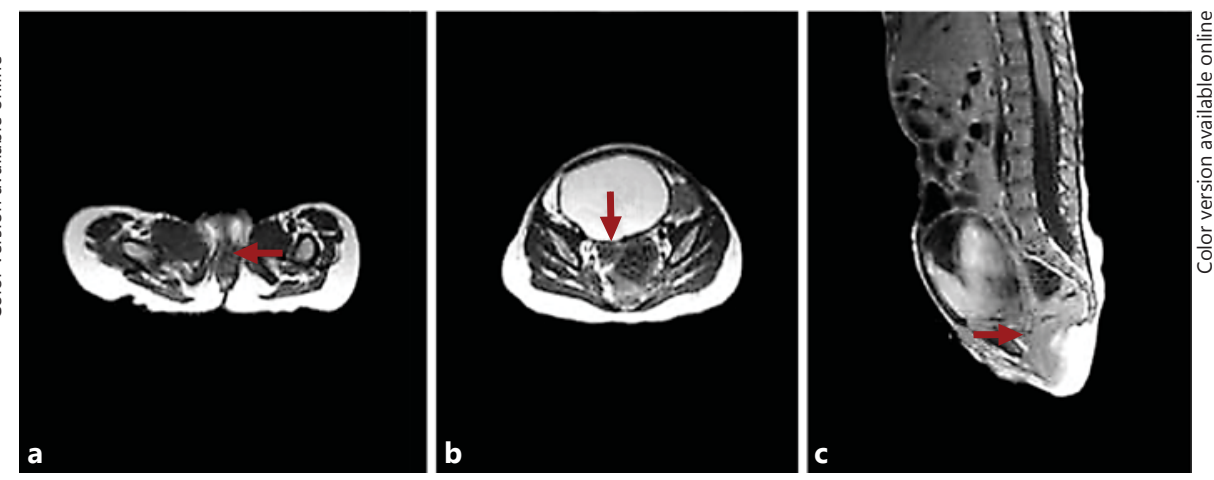

Fig. 2. MRI confirmed the presence of a vagina (a, c) and a uterus (b).

\section{Case Report}

A 3-year-old boy who had been raised male, with facial dimorphism and malformations of both elbows and forearms, was referred to our unit for ambiguous genitalia. The child was the firstborn of a non-consanguineous marriage, delivered at 35 weeks of gestation by cranial presentation with a birth weight of $1,800 \mathrm{~g}$. His medical history was somewhat obscure due to low parental compliance and past referrals to several primary and secondary paediatric centres with no or poor experience in managing complex malformations. A clinical examination carried out upon hospital admission revealed facial dysmorphism: a trapezoidal face, a flat nose and low-set ears; moreover, both elbow joints had severe limitations of flexion-extension and pro-supination movement. X-ray examination of the elbows and forearms revealed bilateral radiohumeral and radioulnar synostosis. Examination of the external genitalia revealed a hypoplastic phallic-like structure with the urethral opening at its base (fig. 1). Below, in the perineum, were labial scrotal folds but they had no obvious content on palpation. Cardiac, renal and gastrointestinal functions were all within the normal range. The endocrine profile showed elevated 17-hydroxyprogesterone and follicle-stimulating hormone, and normal cortisol, DHEA-S, luteinizing hormone and mineralocorticoids. Karyotyping revealed a 46XX pattern. Based on the clinical assessment, the suspicion of ABS was raised. The patient was further referred to the Department of Medical Genetics, where amplification and direct sequencing of all coding exons of the POR gene were performed. The results indicated the homozygous transversion c.859G $>$ C. Both abdominal ultrasound and MRI confirmed the presence of a uterus but failed to reveal ovaries within the pelvis. MRI also revealed a hypoplastic vagina that ended in a distal atretic urogenital sinus (fig. 2). The exact length of the common channel and the exact relation of the vagina and urethra could not be determined from the MRI. Further clinical and paraclinical examinations revealed no other malformations. The patient was seen by a multidisciplinary team composed of a geneticist, an endocrinologist, a paediatric surgeon and a paediatric psychiatrist. Based on the karyotype and an almost normal ovarian function, the team of specialists recommended surgical intervention, which the parents accepted. The surgical intervention for the ambiguous genitalia was performed through a combined perineal and transabdom- inal approach; the external orifice of the urogenital sinus was posteriorly opened and the urethra was identified, isolated and cannulated via a median perineal incision. The urethral meatus was transposed $1.5 \mathrm{~cm}$ posteriorly from the hypertrophied clitoris using part of the common channel to reconstruct the urethra. A median subumbilical incision was performed and the lower peritoneal cavity was entered in order to evaluate the status of the internal genitalia. Inside the peritoneal cavity, the uterus and the two ovaries were identified; both were of normal appearance. A combined sharp and blunt dissection of the vagina was performed and it was mobilized to the perineum in a caudal direction. The distal end of the vagina was identified approximately $1 \mathrm{~cm}$ above the perineal skin. The vagina was approximately $1-1.5 \mathrm{~cm}$ in length and approximately $0.5 \mathrm{~cm}$ in diameter. The perineum trough was opened using a Y-incision, and it anastomosed the mobilized vagina to the perineum. Two lateral incisions were then performed and, by folding the tegument of the pseudoscrotum, the two labia majora were tailored. Finally, the clitoris was folded backward and attached to the perineal skin between the two labia. Because the vaginal neo-introitus had a tendency towards stenosis, vaginal dilatation was performed on a daily basis after the surgery. The patient received prophylactic antibiotics as well as pain and anti-inflammatory medications. The postoperative course was uneventful and the patient was released from the hospital 10 days after the surgery. Six months later, the overall appearance of the external genitalia was satisfactory despite the fact that the reconstructed labia majora were somehow hypoplastic. The vaginal and urethral openings were patent, non-stenotic and clearly demarcated from each other and the patient had good continence for urine and faeces.

\section{Discussion}

The clinical picture of this case comprises several classical features of ABS. The characteristic facial dysmorphism, the synostoses of the elbow bones and the ambiguous genitalia were all present. Interestingly, despite displaying the typical clinical aspect of POR deficiency, the 
patient underwent several medical examinations and was seen by a number of different doctors before the suspicion of ABS was raised. From a medical point of view, the absence of major cardiac, renal or spinal deformities was in the patient's favour and counted towards the decision to carry out the surgical repair of the ambiguous genitalia. In the absence of major associated malformations, the life span of this patient is expected to be good.

The common channel was short. The isolated vagina and urethra were near the perineal tegument and only a short mobilization was required. Nonetheless, mobilization was required and the choice of performing a combined transabdominal and perineal surgical intervention was not dictated only by the need to mobilize the urogenital sinus. The preoperative MRI could not clearly reveal the anatomy of the urogenital sinus or the internal genital structures, so we decided that the best approach was to directly visualize the ovaries.

The most challenging part of the procedure was to create the vaginal introitus and the two labia majora from the pseudoscrotal skin flaps. Because of the excessive skin tension and poor subcutaneous tissue, the reconstructed labia majora had a poor consistency and after a while became hypoplastic. Perhaps this result was due to insufficient folding of the skin and insufficient suturing and fixation of the folded skin flaps to the underlying tissue. We were rather disappointed with the final aspect of the labia majora, and further plastic surgery may resolve this problem.

Another important step as far as reconstructive surgery of ambiguous genitalia is concerned is the clitoroplasty. The surgeon must keep in mind that the clitoris, despite its penis-like appearance, is essential for normal sexual sensations. The most popular method employed nowadays is a reduction clitoroplasty in a dorsal nervesparing fashion by maintaining the glans and resecting parts of the erectile bodies [7]. In our case, we decided not to perform any excision on the clitoris. As the hypertrophy of the clitoris was minimal to moderate, we preferred to simply fold it back and partially bury it in the leftover gap formed by the transposition of the urethra. In this way, we succeeded in obtaining the expected cosmetic results with minimal trauma to the clitoris innervation.

\section{Conclusions}

Repair of the ambiguous genitalia in this patient was possible, but definitive inferences on the benefit of this intervention cannot be made without long-term followup.

\section{References}

1 Antley R, Bixler D: Trapezoidocephaly, midfacial hypoplasia and cartilage abnormalities with multiple synostoses and skeletal fractures. Birth Defects Orig Artic Ser 1975;11: 397-401.

2 Orphanet: Prevalence of rare diseases: bibliographic data - listed in alphabetical order of disease or group of diseases. 2013. http:// www.orpha.net/orphacom/cahiers/docs/GB/ Prevalence_of_rare_diseases_by_alphabetical_list.pdf (accessed November 4, 2012).
3 Orphanet: Antley-Bixler syndrome. 2007. http://www.orpha.net/consor/cgi-bin/OC_ Exp.php?lng=EN\&Expert $=83$ (accessed November 4, 2012).

4 Miller LW: Disorders of androgen synthesis - from cholesterol to dehydroepiandrosterone. Med Princ Pract 2005; 14(suppl 1):5868.

-5 McGlaughlin KL, Witherow H, Dunaway DJ, et al: Spectrum of Antley-Bixler syndrome. J Craniofac Surg 2010;21:1560-1564.

\footnotetext{
Fukami M, Horikawa R, Nagai T, et al: Cytochrome $\mathrm{P} 450$ oxidoreductase gene mutations and Antley-Bixler syndrome with abnormal genitalia and/or impaired steroidogenesis: molecular and clinical studies in 10 patients. J Clin Endocrinol Metab 2005;90:414-426.

$\checkmark 7$ Senaylı A: Controversies on clitoroplasty. Ther Adv Urol 2011;3:273-277.
} 